\title{
Measurement of Surface Orientations of Transparent Objects Using Polarization in Highlight
}

\author{
Megumi Saito $§$ Yoichi Sato $†$ \\ $\S$ Dept. of Electrical Engineering \\ Keio University \\ 3-14-1 Hiyoshi, Kohoku-ku, \\ Yokohama 223-8522, Japan
}

\author{
Katsushi Ikeuchi $\nmid \quad$ Hiroshi Kashiwagi§ \\ $\dagger$ Institute of Industrial Science \\ The University of Tokyo \\ 7-22-1 Roppongi, Minato-ku, \\ Tokyo 106-8558, Japan
}

\begin{abstract}
This paper proposes a method for obtaining surface orientations of transparent objects using polarization in highlight. Since the highlight, the specular component of reflection light from objects, is observed only near the specular direction, it appears merely limited parts on an object surface. In order to obtain orientations of a whole object surface, we employ a spherical extended light source. This paper reports its experimental apparatus, a shape recovery algorithm, and its performance evaluation.
\end{abstract}

\section{Introduction}

Within the optical community, several non-contact optical methods have been proposed for determining surface orientations. These methods can be classified into two classes: point and surface[1]. A point measurement method, which employs optical spots or a beam, scans an entire object surface, measures depth values at each point, and recovers the shape of an object from these measured depth values. A surface measurement method, for example, interferometry or moire topography, illuminates object surfaces with specially designed light sources, analyzes illuminated patterns on the surfaces, and then determines the object shape from this analysis. These methods are effective when applied to a solid surface. Unfortunately, however, no easy methods, with incoherent lights, exist to determine the shapes of transparent objects.

The computer vision community has also developed several methods for determining surface orientations using the amount of reflected light (image brightness). Generally speaking, some component of incoming light is immediately reflected from the object surface. This is referred to as surface reflection. Other components penetrate the object surface, are then inter-reflected among internal pigments of the object, and eventually emitted into the air. This is referred to as body reflection. There is a massive amount of research, referred to as the shape-from-shading method, to determine surface shape by using body reflection.
Relatively little research has been done to utilize surface reflection for determining the object shape. Ikeuchi[2] employs extended light sources that consist of a wide planar surface illuminated by three light sources located at three different positions, takes three images of specular objects under these extended light sources, and determines surface orientations from image triples at each point using the photometric stereo method. Nayar, Ikeuchi, and Kanade[4] extended the method using a spherical diffuser illuminated with many point light sources located around the sphere. Their method determines not only surface orientations but also reflection parameters. Koshikawa[3] measures polarization of surface reflection components under incoherent light sources and determines surface orientations from the degree of polarization. Wolff[9] also proposes to utilize polarization for determining surface orientation. These methods, although having potential, have not been actually applied to determine surface orientations of transparent surfaces.

This paper proposes to use the polarization in surface reflection components for determining shapes of transparent objects. Our method employs the usual incoherent light sources and CCD camera to determine these shapes. This paper describes how we actually set up an experimental apparatus and then evaluates the performance of the method under this apparatus. In Section 2, we review the reflection mechanism. Section 3 describes the algorithm used to determine surface orientations. This section also evaluates the accuracy of the system using simulated images. Section 4 describes the measurement apparatus and its performance. Section 5 concludes the paper.

\subsection{Theory of Reflection and Polarization Light}

A general reflection model is described in terms of two reflection components, namely the body reflection and the surface reflection. In many computer vision and computer graphics applications, reflection models are represented by linear combinations of these two components. This reflec- 
tion model was formally introduced by Shafer[7] as the dicromatic reflection model. We refer to the surface reflection component as the highlight.

Because the surface of transparent objects reveals only the highlight, we propose to utilize polarization of the highlight to obtain the surface shape.

Let us assume that an interface surface between a medium 1 and 2 is located in the $x-y$ plane, as shown in Figure1. Refractive index of a medium 1 and 2 are $n_{1}$ and $n_{2}$, respectively. The light wave in the $x-z$ plane hits the interface surface; one part of the light is reflected at the origin, the other part is refracted and transmitted into the medium 2. Here we assume that the object consists of transparent dielectric materials so that the absorption can be ignored when considering visible light.

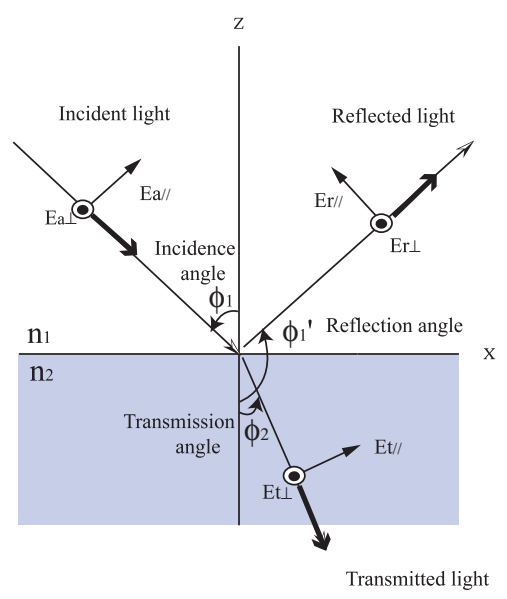

Figure 1: The Fresnel reflection

The polarization components of incident, reflected, and transmitted light, parallel or perpendicular to the $x-z$ plane, are expressed by subscript $\|$ or $\perp$, respectively. We define incidence angle $\phi_{1}$, reflection angle $\phi_{1}^{\prime}$, and transmission angle $\phi_{2}$ as depicted in Figure 1. Incident and reflected light go through the same medium. As a result of this, we get $\phi_{1}=\pi-\phi_{1}^{\prime}$. The incident, reflected, and transmitted components of the electric field vector parallel to the $x-z$ plane, $E_{a \|}, E_{r \|}$, and $E_{t \|}$ are

$$
\begin{aligned}
E_{a \|} & =A_{\|} \exp \left[i\left\{\omega t-k_{1}\left(x \sin \phi_{1}+z \cos \phi_{1}\right)\right\}\right] \\
E_{r \|} & =R_{\|} \exp \left[i\left\{\omega t-k_{1}\left(x \sin \phi_{1}-z \cos \phi_{1}\right)\right\}\right](1) \\
E_{t \|} & =T_{\|} \exp \left[i\left\{\omega t-k_{2}\left(x \sin \phi_{2}+z \cos \phi_{2}\right)\right\}\right]
\end{aligned}
$$

where $A_{\|}, R_{\|}$, and $T_{\|}$represent amplitudes of the three components, $\omega$ represents angular frequency, and $k_{1}$ and $k_{2}$ represent the wave number, respectively, where the wave number is expressed as $2 \pi / \lambda . \quad \lambda$ is the wavelength. Subscripts a, r, and t denote incident, reflected, and transmitted light, respectively. The perpendicular component is ex- pressed in a similar manner. The relation between incidence angle, $\phi_{1}$ and transmission angle, $\phi_{2}$ of the refracted light, when penetrating from one medium to another, is given by Snell's law:

$$
n_{1} \sin \phi_{1}=n_{2} \sin \phi_{2} \text {. }
$$

The boundary condition of the Maxwell equation requires that components of electric and magnetic field on boundary plane must be continuous at the plane. Thus, the amplitude of the transmitted light in the medium 2 must be equivalent to the sum of the amplitude of the incident light and one of the reflected lights in the medium 1 in the $x$ and $y$ direction. From this, we obtain

$$
E_{a j}+E_{r j}=E_{t j}, \quad H_{a j}+H_{r j}=H_{t j} \quad(j=x, y)
$$

where $\mathrm{E}$ and $\mathrm{H}$ denote electric and magnetic fields, respectively. This can be combined with equation(1) and (2) to yield the Fresnel formula that expresses reflectance of light amplitude with respect to the parallel and perpendicular components, $r_{\|}$and $r_{\perp}$.

$$
\begin{aligned}
& r_{\|}=\frac{E_{r \|}}{E_{a \|}}=\frac{\tan \left(\phi_{1}-\phi_{2}\right)}{\tan \left(\phi_{1}+\phi_{2}\right)} \\
& r_{\perp}=\frac{E_{r \perp}}{E_{a \perp}}=-\frac{\sin \left(\phi_{1}-\phi_{2}\right)}{\sin \left(\phi_{1}+\phi_{2}\right)}
\end{aligned}
$$

Image intensity, $I$ is expressed as

$$
I=\frac{n E^{2}}{2 \sqrt{\mu_{0}}}
$$

where $n$ stands for a refractive index of each medium and $\mu_{0}$ is permeability in vacuum. Likewise, from equation (4), reflectance of light intensity is given by

$$
\begin{aligned}
F_{\|} & =\frac{\tan ^{2}\left(\phi_{1}-\phi_{2}\right)}{\tan ^{2}\left(\phi_{1}+\phi_{2}\right)} \\
F_{\perp} & =\frac{\sin ^{2}\left(\phi_{1}-\phi_{2}\right)}{\sin ^{2}\left(\phi_{1}+\phi_{2}\right)}
\end{aligned}
$$

Intensity reflectance $F_{\|}, F_{\perp}$ are referred to as the Fresnel reflection coefficients. Equation (6) indicates that there is an angle of incidence which yields $F_{\|}=0$. This angle of incidence is referred to as the Brewster angle $\phi_{b}$. The Brewster angle is given by $\phi_{1}+\phi_{2}=\pi / 2$ and the Snell's law by

$$
\phi_{b}=\arctan \left(\frac{n_{2}}{n_{1}}\right)
$$

\section{Measurement of Surface Orientations of Transparent Objects \\ 2.1 Theory of Measurement}

Usually, natural light is unpolarized; it oscillates randomly in all directions. Sometimes such natural light is 
polarized, for example, when transmitted through a birefringent crystal or when reflected from an object surface. When part of the light is polarized, that portion is called partial polarized light. Here we study reflection from objects. Reflected light is the sum of the body and the surface reflection components. The body reflection component of reflected light is ordinarily unpolarized. However, it can be ignored in transparent objects.

We will determine the surface normal from the direction of polarization in the surface component. When light waves, transverse electromagnetic waves, propagate along one direction, the electric field vector lies approximately on a plane perpendicular to the transverse line. The electric and magnetic wave oscillates on the plane and those oscillations may be biased, polarized, in a certain direction. This polarization depends on the incidence angle and the orientation of the plane of incidence.

The geometry of our measurement system is shown in Figure2. We define the plane of incidence as the one that includes the direction of a light source, a viewer, and a surface normal. In transparent objects, the body reflection component can be ignored and absorption does not occur. The reflection angle is equal to the incidence angle. We can obtain surface normal orientations by using orientation of the plane of incidence and reflection angle at each point of the objects surface. We denote the orientation of the plane with $\theta$ measured around the viewer's line of sight, and denote the angle of incidence with $\phi$ measured on the plane of incidence.

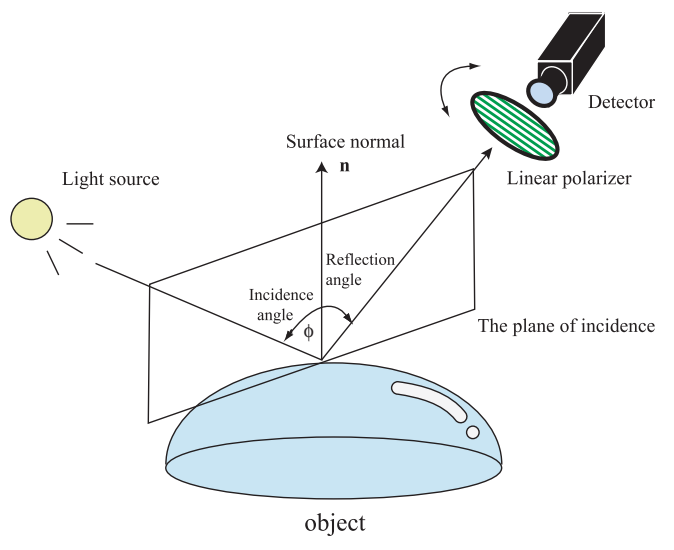

Figure 2: Surface normal of object

As seen in equation (6), intensity reflectance depends on a direction of a plane of oscillation, parallel or perpendicular. Measured brightness changes when a polarizer is rotated in front of a detector. We define the maximum and minimum of the light intensity as $I_{\max }$ and $I_{\min }$, respectively. The sum of $I_{\max }$ and $I_{\min }$ is equal to the total light intensity of the surface component, $I_{s}$.

$$
I_{\max }=\frac{F_{\perp}}{F_{\|}+F_{\perp}} I_{s}, \quad I_{\min }=\frac{F_{\|}}{F_{\|}+F_{\perp}} I_{s}
$$

Since $I_{\min }$ is the component parallel to the plane of incidence, the orientation of the plane of incidence $\theta$ can be determined when $I_{\min }$ appears while rotating the polarizer.

We define the degree of polarization, $\rho$ as:

$$
\rho=\frac{I_{\max }-I_{\min }}{I_{\max }+I_{\min }}
$$

The degree of polarization is 0 for unpolarized light and 1 for linearly polarized light. When the incidence angle is equal to the Brewster angle, only the perpendicular component appears in the reflected light; the reflected light is linearly polarized, and the degree of polarization is 1 . Substituting equations(6)(8) for (9), consider the Snell's law; the degree of polarization, $\rho$ is given by

$$
\rho=\frac{2 \sin \phi \tan \phi \sqrt{n^{2}-\sin ^{2} \phi}}{n^{2}-\sin ^{2} \phi+\sin ^{2} \phi \tan ^{2} \phi}
$$

The degree of polarization, rho is a function of the angle of incidence, $\phi$ under a given refractive index $n$. Thus, from the measured the degree of polarization, we can obtain the angle of incidence $\phi$ from equation(10).

We can summarize the measurement algorithm as follows:

1. While rotating the polarizer, we measure the light intensity to find the $I_{\max }$ and $I_{\min }$ at each pixel.

2. From equation (9), the parallel component to the plane of incidence is $I_{\text {min }}$. Thus, by finding the polarizer rotation angle that provides the minimum intensity, we can obtain the angle of the plane, $\theta$.

3. The degree of polarization is given by equation (8) with measured $I_{\max }$ and $I_{\min }$.

4. Equation (10) provides the degree of polarization from the refractive index, $n$ and the incidence angle, $\phi$. By inversely solving the equation from a given refractive index and measuring the degree of polarization, we can obtain the incidence angle.

\subsection{Evaluation of Accuracy}

This section evaluates the accuracy of the system. We will measure the deviation of the orientation of the plane of incidence and the angle of incidence depending on errors in measured light intensity.

First, we fix the refractive index $n$. We also choose the angle of incidence, ${ }_{p} h i$ and the orientation of the incidence plane, $\theta$. From these two values, we can obtain the maximum, $I_{\max }$ and the minimum, $I_{\min }$ of the light intensity. 
Then, Gaussian noise is added to these $I_{\max }$ and $I_{\min }$. The angle of incidence $\phi$ and the orientation of incidence plane, $\theta$ are inversely calculated by using these two values and the difference between the real and estimated values of incident angle and the orientation of the incidence plane. This process is repeated 1000 times to obtain the standard deviations of these two angles.

Figure3(a)(b) show the dependence on the error rate of intensity, of the standard deviation of $\theta$ and $\phi$. Here, the refractive index is fixed at 1.5. The change of the standard deviation as a function of the angle of incidence appears in Figure 3(c). The error rate of intensity is fixed at $10 \%$.

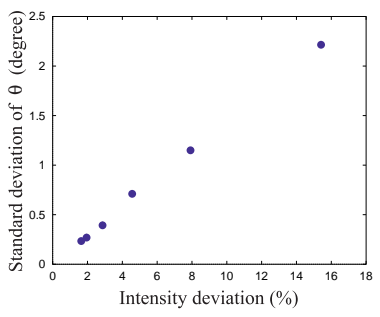

(a)

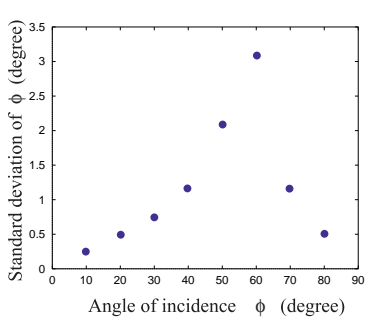

(c)

Figure 3: Simulation result

From this evaluation, we can conclude that the error of the orientation of incidence is 2.2 degrees, while that of the angle of incidence is 1.1 degree at $15 \%$ error range in intensity measurement. This error is linearly proportional to the error in intensity, and thus, we can control the error range by maintaining one in intensity in a lower range. In particular, since the intensity error is affected by the sampling with 8-bit resolution, we can reduce intensity error by illuminating objects brightly enough so as to make intensity values larger. As shown in the figure corresponding to the angle of incidence, when the error rate is fixed to $10 \%$, the standard deviation of its error has as its maximum, about 3.1 degrees, at 60 degrees. Since the Brewster angle is 56.3 degrees, the measurement system is most affected by noise in the vicinity of the Brewster angle.

\subsection{Experimental Method}

We conducted an experiment on measuring a transparent object shape. A white $150 \mathrm{~W}$ incandescent electric lamp was used as the light source. As an object for measuring, we employed a 30-mm X 30-mm X 3-mm plane glass plate, which consisted of soda-lime glass whose refractive index was 1.523 . And we used a rotary stage to position the object in an arbitrary direction. A monochromatic CCD camera was used as a reflected light detector. The image of the CCD had 480 X 512 pixels and each pixel had 8 bits, that is, 256 gradations.

The approach employed in this experiment was as follows. We repeatedly took images by the CCD in order as we rotated the polarizer in front of the camera at an angle from 0 degrees through 180 degrees at intervals of 5 degrees. The result was that we obtained 36 images. Variance in intensity of the light at each pixel could be observed in the images. By using the variations, we were able to obtain the maximum and minimum of intensity. Since a 5 degree sampling interval of a polarized angle may not be so small, a discrepancy between experimental data and actual value of the maximum and minimum may have occurred. In order to avoid this, we fitted a sinusoid function to the experimental approach using the nonlinear root mean square method and estimated the actual maximum and minimum values.

As described in Section 3.1, surface normal orientations can be calculated by $I_{\max }$ and $I_{\min }$. We conducted an experiment on rotating the object at a reflection angle from 10 degrees to 80 degrees at intervals of 10 degrees. The orientation of the plane of incidence was fixed at 90 degrees. We employed an algorithm using the reflection theory and calculated surface orientations based on experimental data at each pixel. Here, it could be presumed that the lens used in this experiment had a focal length long enough that its projection geometry can be approximated as the orthographic projection. It is appropriate that all results at each pixel were equivalent because the object had a plane surface. Therefore we calculated the average and the standard deviation of the orientations of the plane of incidence and the angles of incidence obtained at all pixels, respectively. The average is used as the result of this experiment.

\subsection{Experimental Results}

The results of the orientation of the incident plane, $\theta$ and the angle of incidence, $\phi$ obtained experimentally are depicted, as a function of $\phi$, in Figure4(a) and Figure4(b), respectively. In Figure4(a), the obtained orientations of the incident plane are very close to the real values, 90 degrees depicted with the dotted line except around 10 degrees of incident angle, while, in Figure4(b), the obtained incident angles are close to the real data. At small incident angles, the degree of polarization is also small, and the amplitude of the sinusoidal function is relatively smaller than other areas; fitting errors of the non-linear minimization become larger. We can, however, improve this by increasing the brightness of the images, thereby resulting in a larger amplitude of the function. 


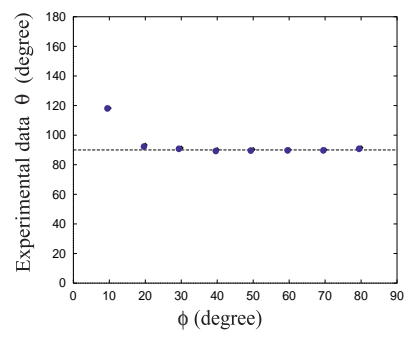

(a) The orientation of the plane of incidence

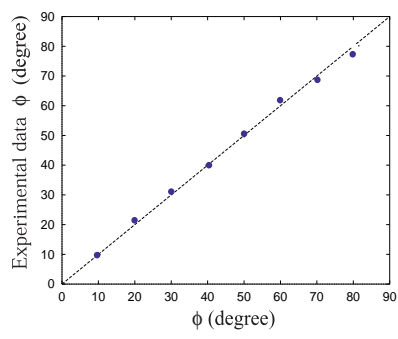

(b) The angle of incidence
Figure 4: Experimental result

\section{Measurement by Using a Spherical Ex- tended Light Source}

\subsection{Theory of Measurement}

The previous section evaluated the accuracy of the proposed algorithm, and demonstrated its effectiveness. This section proposes a practical measurement system that employs an extended light source. As stated previously, however, the highlight is observed only near the specular direction; and thus, if we use an ordinal point light source, we can obtain surface orientations only at relatively limited areas that satisfy the specular geometry. To overcome this problem, we employ a spherical extended light source.

An extended light source emits light from a region with a certain area size. This is in contrast to a point light source, such as an electric lamp, that emits spherical light wave from one point, without an area size, to surrounding directions. The highlight depends on the direction of the light source and viewing direction but it becomes possible to reduce the dependence on the direction of the light source by using an extended light source. In particular, a spherical extended light source, where we put an object in the center of the sphere, can project light to the object from all directions. The highlight can be generated on the entire surface of the object. It becomes possible to obtain orientations of the entire surface without scanning the camera.

The experimental apparatus on the spherical extended light source is depicted in Figure5. An optical diffuser of a white translucent plastic sphere whose diameter is $40 \mathrm{~cm}$, for the spherical extended light source, is illuminated using three incandescent electric lamps placed at intervals of 120 degrees. An object is placed in the center of this sphere. Using a CCD camera, images of the object are taken through a hole located at the north pole of the sphere.

\subsection{Experimental Results}

We conducted an experiment on measuring an object, a plastic hollow hemisphere with refractive index 1.55 whose diameter is $6 \mathrm{~cm}$. In the same way as described in Section 3, we repeatedly took images in order as we rotated the polarizer in front of the CCD camera. Figure6(a) depicts

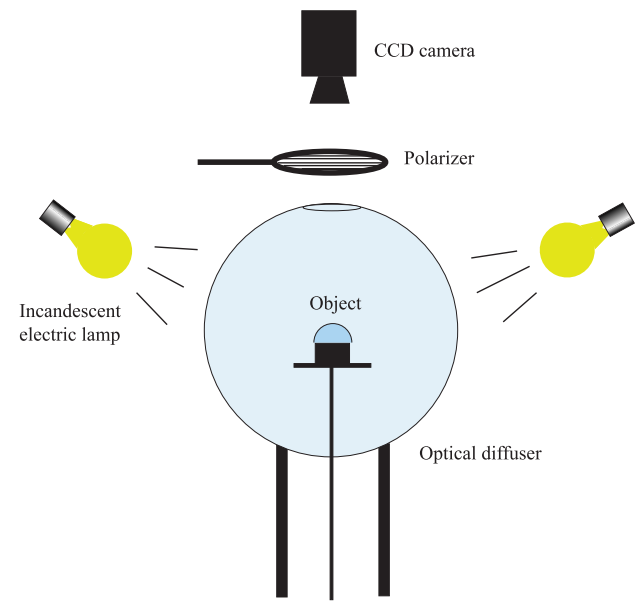

Figure 5: Experimental setup

obtained surface normals as needles, displayed at every 20 pixels. From the figure, the directions of needles are rotationally symmetrical with respect to the center of the object, and their lengths increase along the radius direction of the object. We concluded that the obtained surface normalizations are consistent with those of the object. Figure6(b) shows the calculated object shape from those obtained surface normals for visualization.

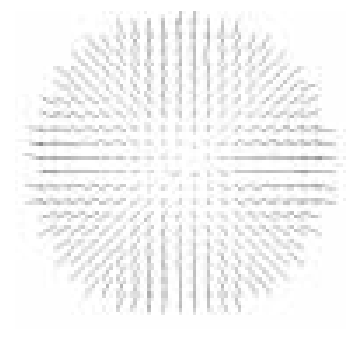

(a)

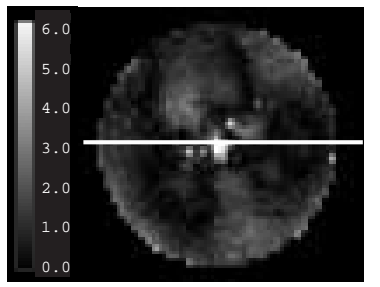

(c)

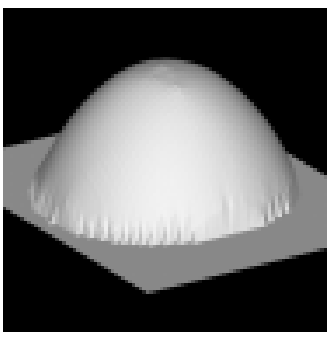

(b)

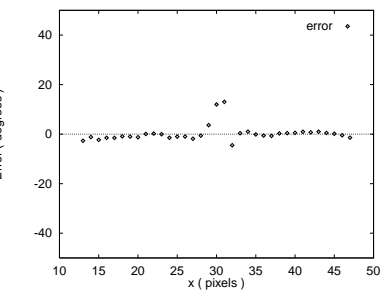

(d)
Figure 6: Result of measurement of the object shape

Figure6(c) visualizes the difference between the estimated angle of incidence and the real angle of incidence. In this figure, a brighter pixel represents larger error. The region where angle of incidence is more than 50 degrees 
was excluded in Figure6(c) since the region is too close to the occluding boundary of the hemisphere.

Figure6(d) shows the plot of the error in angle of incidence along the scan line drawn in Figure6(c). From these results, we can see that the error becomes significantly large at small angle of incidence, while the error is small otherwise. This is because the spherical extended light source used in our experiments has a hole through which the camera sees the object. Due to this hole, the light source does not cover the region of small angle of incidence. The average error in angle of incidence was 0.82 degrees in this result.

For the next experiment, we demonstrated the ability of the system to detect flaws on transparent objects. We made a flaw whose width was about 100 micro meters, on the glass plate used in Section 3 and measured surface normals using the same procedure. Figure7 shows this result. The black region on the result image depicts one consisting of pixels with normals that deviate from the average orientation.

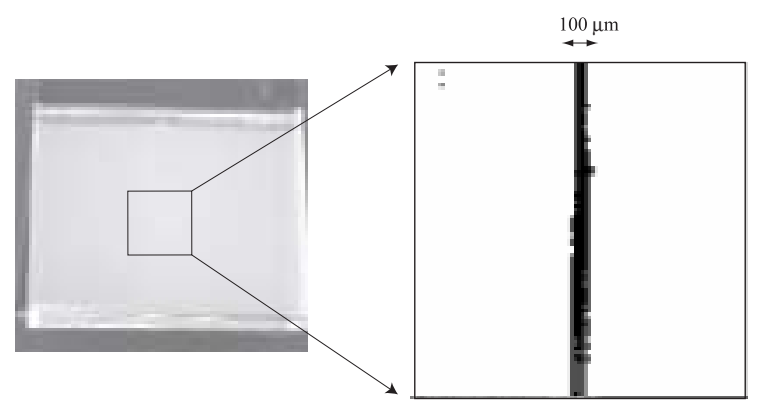

Figure 7: Result of flaw inspection

\section{Conclusion}

This paper proposed a method for measuring surface orientations of transparent objects using polarization in highlight. The paper also demonstrated the methods effectiveness and evaluated its accuracy by describing experiments performed using synthesized and real images. We also proposed employing a spherical extended light source for the system. This is because the highlight, the specular of reflection light from objects, is observed only near the specular direction and appears on relatively limited parts on an object surface if we use an ordinal point light source. The experimental results show that the obtained surface orientations are highly accurate, and that the proposed method is effective. We also demonstrated the ability of the system to detect flaws of transparent objects and the potential for practical shape inspection applications of the system.

\section{References}

[1] T.Tanitagai, Applied Optics: Guide of Optical Measurement, Maruzen, 1988.

[2] K.Ikeuchi "Determining surface orientations of specular surfaces by using the photometric stereo method," IEEE Trans. Patt. Anal. Mach. Intell., Vol. 3, pp. 661669, 1981.

[3] K.Koshikawa "A polarimetric approach to shape understanding of glossy objects," Proceedings of IJCAI, pp. 493-495, 1979.

[4] S.K.Nayar, K.Ikeuchi, and T.Kanade "Determining shape and reflectance of hybrid surface by photometric sampling," IEEE Trans. Rob. Auto., Vol. 6, pp. 418-431, 1990.

[5] S.K.Nayar, K.Ikeuchi, T.Kanade "Surface reflection: Physical and geometrical perspectives," IEEE Trans. Patt. Anal. Mach. Intell., Vol. 13, pp. 611-634, 1991.

[6] M.Ohtsu, Modern Optical Science, Asakura, 1994.

[7] S.Shafer, "Using color to separate reflection components," COLOR Research and Application, Vol. 10, pp. 210-218, 1985.

[8] K.E.Torrance, and E.M.Sparrow "Theory for offspecular reflection from roughened surfaces," J. Opt. Soc. Am., Vol. 57, pp. 1105-1114, 1967.

[9] L.B.Wolff "Spectral and polarization stereo methods using a single light source," Proceedings, ICCV, pp. 708-715, 1987.

[10] L.B.Wolff, T.E.Boult "Constraining object features using a polarization reflectance model," IEEE Trans. Patt. Anal. Mach. Intell., Vol. 13, pp. 167-189, 1991. 